\title{
TUMORES MALIGNOS DA CAVIDADE NASAL: TOMOGRAFIA COMPUTADORIZADA E RESSONÂNCIA MAGNÉTICA*
}

\author{
Ricardo Pires de Souza ${ }^{1}$, Ademar José de Oliveira Paes Junior ${ }^{2}$, Carlos Neutzling Lenh ${ }^{3}$, \\ Fábio Mota Gonzalez ${ }^{2}$, Flamarion de Barros Cordeiro ${ }^{2}$, Ilka Yamashiro ${ }^{2}$, Abrão Rapoport ${ }^{4}$
}

Resumo OBJETIVO: Este estudo propõe-se a avaliar o papel da tomografia computadorizada e da ressonância magnética na caracterização da extensão profunda dos tumores malignos da cavidade nasal. MATERIAIS E MÉTODOS: Entre 1990 e 2000 foram avaliados, retrospectivamente, 12 pacientes com diagnóstico de tumores malignos da cavidade nasal atendidos nos Departamentos de Diagnóstico por Imagem e Cirurgia de Cabeça e Pescoço do Complexo Hospitalar Heliópolis, São Paulo, SP. Todos os casos foram confirmados com exame anatomopatológico. RESULTADOS: Foi identificada extensão para os seios maxilares e etmoidal em seis pacientes, para a cavidade nasal contralateral, órbita e lâmina crivosa em cinco pacientes, para a nasofaringe e espaço mastigatório em dois pacientes, e para o seio cavernoso, fossas cranianas anterior e média, fossa pterigomaxilar, fissuras orbitárias superior e inferior, seio frontal, seio etmoidal contralateral, lâmina crivosa contralateral, palato duro e fossa pterigopalatina em um paciente. CONCLUSÃO: A análise precisa da extensão local e disseminação tumoral dada pela tomografia computadorizada e ressonância magnética desempenha papel importante no planejamento terapêutico, influenciando também o prognóstico. Unitermos: Cavidade nasal; Neoplasia maligna; Tomografia computadorizada.

Abstract Malignant tumors of the nasal cavity: computed tomography and magnetic resonance imaging.

OBJECTIVE: The aim of this study is to evaluate the role of computed tomography and magnetic resonance imaging in the characterization of deep tissue extension of malignant tumors of the nasal cavity. MATERIALS AND METHODS: Twelve patients diagnosed with malignant tumors of the nasal cavity were retrospectively evaluated at the Departments of Diagnostic Imaging and Head and Neck Surgery of the "Complexo Hospitalar Heliópolis", São Paulo, Brazil, between 1990 and 2000. All cases were confirmed by histopathologic examination. RESULTS: Extension to the maxillary and ethmoid sinuses was identified in six patients, extension to contralateral nasal cavity, orbit and lamina cribosa in five patients, extension to nasal pharynx and masticator space in two patients, extension to cavernous sinus, anterior/middle cranial fossa, pterygomaxillary fossa, inferior/superior orbital fissure, frontal sinus, contralateral ethmoid sinus, contralateral lamina cribosa, hard palate and pterygopalatine fossa in one patient. CONCLUSION: It is important to precisely assess the local extension and spread of tumor by computed tomography and magnetic resonance imaging in order to plan the approach to treatment, which will influence the prognosis.

Key words: Nasal cavity; Malignant neoplasm; Computed tomography; Magnetic resonance imaging.

\section{INTRODUÇÃO}

Os tumores malignos nasossinusais compreendem $0,2 \%$ a $0,8 \%$ de todos os tumores malignos do corpo humano e $3,0 \%$ dos tumores envolvendo o trato aero-

* Trabalho realizado no Serviço de Diagnóstico por Imagem do Complexo Hospitalar Heliópolis, São Paulo, SP.

1. Coordenador do Programa de Residência Médica em Radiologia e Diagnóstico por Imagem do Complexo Hospitalar Heliópolis.

2. Médicos Residentes em Radiologia e Diagnóstico por Imagem do Complexo Hospitalar Heliópolis, Mestrandos do Curso de Pós-Graduação em Ciências da Saúde do Hospital Heliópolis.

3. Chefe do Serviço de Cirurgia de Cabeça e Pescoço do Complexo Hospitalar Heliópolis.

4. Coordenador do Curso de Pós-Graduação em Ciências da Saúde do Hospital Heliópolis.

Endereço para correspondência: Dr. Ricardo Pires de Souza. Rua Cônego Xavier, 276, 10aandar, Bairro Sacomã. São Paulo, SP, 04231-010. E-mail: ricapires @ig.com.br

Recebido para publicação em 3/9/2003. Aceito, após revisão, em 24/11/2003. digestivo superior. Aproximadamente 50\% dos tumores nasossinusais surgem a partir do seio maxilar e cerca de $15 \%$ a $30 \%$, a partir da cavidade nasal. Tumores malignos da cavidade nasal são relativamente raros e esta região é um local de tipos variados de neoplasias, a maioria delas de origem epitelial, dos quais os carcinomas representam $50 \%$ das lesões ${ }^{(\mathbf{1}, 2)}$.

Clinicamente, os sinais e sintomas desses tumores podem ser indistinguíveis das lesões benignas e processos inflamatórios. Os sintomas nasais são, inicialmente, obstrução nasal, coriza e epistaxe. Nos casos mais avançados, erosão do palato, invasão da órbita ou intracraniana podem ocorrer, surgindo sintomas como hiperestesia, neuropatia dos nervos cranianos, edema facial, trismo, distúrbios visuais e proptose. Após realizar o exame clínico, a investigação dos tumores malignos prossegue através de exames de imagem, preferencialmente tomografia computadorizada (TC) e ressonância magnética (RM). Estes exames são necessários para identificar a topografia da lesão e definir a sua extensão local ${ }^{(3,4)}$.

Este estudo propõe-se a avaliar o papel da TC e RM na caracterização da extensão profunda dos tumores malignos da cavidade nasal diagnosticados em 12 pacientes, atendidos entre 1990 e 2000.

\section{MATERIAIS E MÉTODOS}

A amostra de 12 pacientes com neoplasia maligna da cavidade nasal, atendidos nos Departamento de Diagnóstico por Imagem e Cirurgia de Cabeça e Pescoço do 
Complexo Hospitalar Heliópolis, São Paulo, SP, entre 1990 e 2000, foi analisada de forma retrospectiva neste estudo.

Todos os pacientes foram submetidos a biópsia e os diagnósticos foram confirmados por exame anatomopatológico. Foram encontrados três casos de estesioneuroblastoma ( $25 \%)$, dois casos de carcinoma espinocelular $(16,8 \%)$, dois casos de melanoma $(16,8 \%)$, um caso de linfoma $(8,3 \%)$, um caso de rabdomiossarcoma $(8,3 \%)$, um caso de adenocarcinoma $(8,3 \%)$, um caso de carcinoma de células transicionais $(8,3 \%)$ e um caso de carcinoma indiferenciado $(8,3 \%)$, conforme mostra a Tabela 1 .

Tabela 1 Amostra de 12 pacientes com tumores malignos da cavidade nasal.

\begin{tabular}{|l|r|r|}
\hline \multicolumn{1}{|c|}{ Histologia } & $\mathrm{N}$ & $\%$ \\
\hline Estesioneuroblastoma & 3 & $25,0 \%$ \\
Carcinoma espinocelular & 2 & $16,8 \%$ \\
Melanoma & 2 & $16,8 \%$ \\
Linfoma & 1 & $8,3 \%$ \\
Rabdomiossarcoma & 1 & $8,3 \%$ \\
Adenocarcinoma & 1 & $8,3 \%$ \\
Carcinoma células transicionais & 1 & $8,3 \%$ \\
Carcinoma indiferenciado & 1 & $8,3 \%$ \\
\hline Total & 12 & $100 \%$ \\
\hline
\end{tabular}

A distribuição por sexo foi de sete mulheres $(58,3 \%)$ e cinco homens $(41,7 \%)$, com idade variando entre 16 e 76 anos (idade média de 54 anos). Cinco pacientes eram fumantes $(41,7 \%)$ e três relataram história prévia de etilismo (Tabela 2).

Até o momento da realização do exame de imagem, 11 pacientes $(91,7 \%)$ não haviam realizado nenhum tipo de tratamento e apenas um fora submetido a quimioterapia. A comparação com os achados cirúrgicos foi possível em sete casos.

Todos os pacientes foram examinados por meio de exames de TC, sendo realizados cortes axiais e coronais contínuos de $5 \mathrm{~mm}$ de espessura e incremento, após a injeção endovenosa do meio de contraste iodado. Três pacientes realizaram RM em aparelhos de 0,5 T e 1,5 T, sendo obtidas imagens ponderadas em T1 e T2 nos planos axiais, coronais e sagitais.

As imagens obtidas por TC e RM dos pacientes submetidos ao estudo foram analisadas, retrospectivamente, por um radiologista com experiência em doenças de

Tabela 2 Informações clínicas de 12 pacientes com tumores malignos da cavidade nasal.

\begin{tabular}{|c|c|c|c|c|c|}
\hline \multirow{2}{*}{ Histologia } & \multirow{2}{*}{$\mathrm{N}$} & \multicolumn{2}{|c|}{ Sexo } & \multirow{2}{*}{ Tabagismo } & \multirow{2}{*}{ Etilismo } \\
\hline & & Masculino & Feminino & & \\
\hline Estesioneuroblastoma & 3 & - & 3 & - & - \\
\hline Carcinoma espinocelular & 2 & 2 & - & 2 & - \\
\hline Melanoma & 2 & 1 & 1 & 1 & 1 \\
\hline Linfoma & 1 & 1 & - & 1 & 1 \\
\hline Rabdomiossarcoma & 1 & 一 & 1 & 一 & - \\
\hline Adenocarcinoma & 1 & 1 & 一 & 1 & 1 \\
\hline Carcinoma células transicionais & 1 & - & 1 & - & - \\
\hline Carcinoma indiferenciado & 1 & - & 1 & 一 & - \\
\hline Total & 12 & 5 & 7 & 5 & 3 \\
\hline
\end{tabular}

cabeça e pescoço, avaliando o sítio primário do tumor e sua extensão para os seios maxilares, seios etmoidais, seio frontal, cavidade nasal contralateral, órbita, lâmina crivosa do etmóide, nasofaringe, espaço mastigador, seio cavernoso, fossa craniana anterior e medial, fossas pterigomaxilar e pterigopalatina e palato duro.

\section{RESULTADOS}

Após determinar-se o sítio primário do tumor, foi definida sua extensão para os seios maxilares e etmoidal em seis pacientes $(50 \%)$, para a cavidade nasal contralateral, órbita e lâmina crivosa do etmóide em cinco pacientes $(41,7 \%)$, para a nasofaringe e espaço mastigatório em dois pacientes $(16,6 \%)$, e para o seio cavernoso, fossas cranianas anterior e média, fossa pterigomaxilar, fissuras orbitárias superior e inferior, seio frontal, seio etmoidal contralateral, lâmina crivosa contralateral, palato duro e fossa pterigopalatina, todos esses em um paciente $(8,3 \%)$, conforme mostra a Tabela 3. Em três pacientes a neoplasia foi caracterizada como confinada à cavidade nasal avaliada (25\%).

\section{DISCUSSÃO}

Os tumores malignos da cavidade nasal somam uma pequena porcentagem de todas as neoplasias malignas de cabeça e pescoço, representando cerca de 3\%. Desses tumores, $50 \%$ a $60 \%$ são carcinomas espinocelulares. Os carcinomas espinocelulares afetam, principalmente, homens (proporção de 2:1 homem:mulher), entre a quinta e a sétima décadas de vida, e es-
Tabela 3 Extensão da doença na avaliação por imagem.

\begin{tabular}{|l|l|}
\hline \multicolumn{1}{|c|}{ Extensão } & Incidência (\%) \\
\hline Seios maxilares & $6 / 12(50,0 \%)$ \\
Seio etmoidal & $6 / 12(50,0 \%)$ \\
Cavidade nasal contralateral & $5 / 12(41,7 \%)$ \\
Órbita & $5 / 12(41,7 \%)$ \\
Lâmina crivosa & $5 / 12(41,7 \%)$ \\
Nasofaringe & $2 / 12(16,6 \%)$ \\
Espaço mastigatório & $2 / 12(16,6 \%)$ \\
Seio cavernoso & $1 / 12(8,3 \%)$ \\
Fossa craniana anterior/média & $1 / 12(8,3 \%)$ \\
Fossa pterigomaxilar & $1 / 12(8,3 \%)$ \\
Fissura orbitária superior & $1 / 12(8,3 \%)$ \\
Fissura orbitária inferior & $1 / 12(8,3 \%)$ \\
Seio frontal & $1 / 12(8,3 \%)$ \\
Seio etmoidal contralateral & $1 / 12(8,3 \%)$ \\
Lâmina crivosa contralateral & $1 / 12(8,3 \%)$ \\
Palato duro & $1 / 12(8,3 \%)$ \\
Fossa pterigopalatina & $1 / 12(8,3 \%)$ \\
\hline
\end{tabular}

tão associados com fatores de risco que incluem a associação entre o consumo de tabaco e de álcool ${ }^{(\mathbf{4 , 5})}$. Neste estudo, a forma mais comumente encontrada de neoplasias malignas da cavidade nasal foi o estesioneuroblastoma (tumor originado do neuroepitélio olfatório), representando $25 \%$ dos casos, seguido pelo carcinoma espinocelular e melanoma, ambos com $16,8 \%$. Os menos comuns, com apenas um caso $(8,3 \%)$ cada, foram: adenocarcinoma, rabdomiossarcoma, linfoma, carcinoma de células transicionais e carcinoma de células indiferenciadas. Essa diferença em relação à literatura pode ser explicada pelo fato de o Departamento de Cirurgia de Cabeça e Pescoço do Complexo Hospitalar Heliópolis ser um serviço de referência. 
Figura 1. Tomografia computadorizada com janela para partes moles, nos planos axial (A) e coronal (B), evidencia massa na fossa nasal direita com densidade heterogênea e aspecto infiltrativo estendendose para os seios etmoidal e maxilar ipsilaterais. Carcinoma espinocelular.

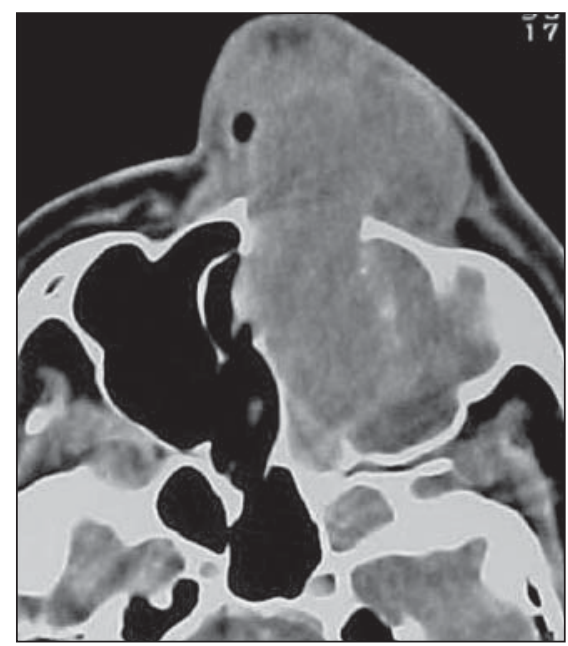

Figura 2. Tomografia computadorizada com janela para partes moles, no plano axial, mostra lesão infiltrativa e heterogênea da fossa nasal esquerda estendendo-se para o seio maxilar ipsilateral, condicionando destruição das paredes ósseas medial e anterior. A massa ocupa a região subcutânea da face. Adenocarcinoma.

O sexo predominante foi o feminino, na proporção de 1,4:1.

A análise crítica, quanto aos tumores da cavidade nasal, consiste na sua natureza (benigno versus maligno), histologia e extensão superficial e profunda. A extensão e disseminação desses tumores têm papel importante no planejamento terapêutico e influencia o prognóstico. $\mathrm{O}$ exame endoscópico promove um estadiamento preciso da disseminação superficial, uma vez que a forma inicial dessas lesões é, geralmente, ulcerada ou polipóide ${ }^{(6,7)}$.

A avaliação pelo radiologista é essencial nas neoplasias malignas. As taxas de precisão para TC quanto à extensão das neoplasias varia entre $78 \%$ e $85 \%$, segundo

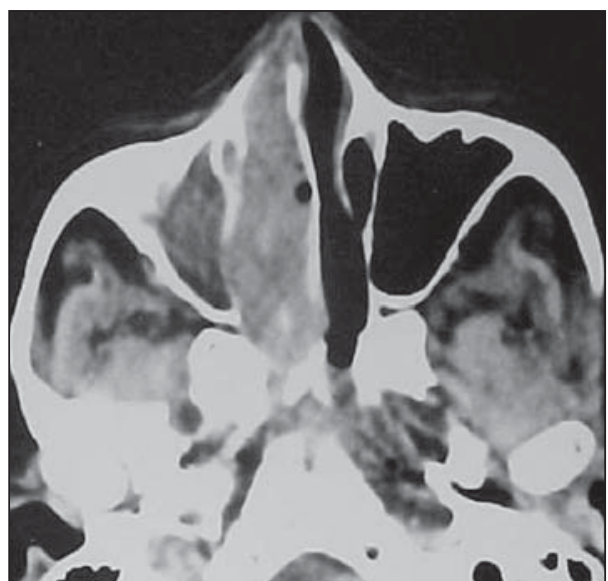

A



A

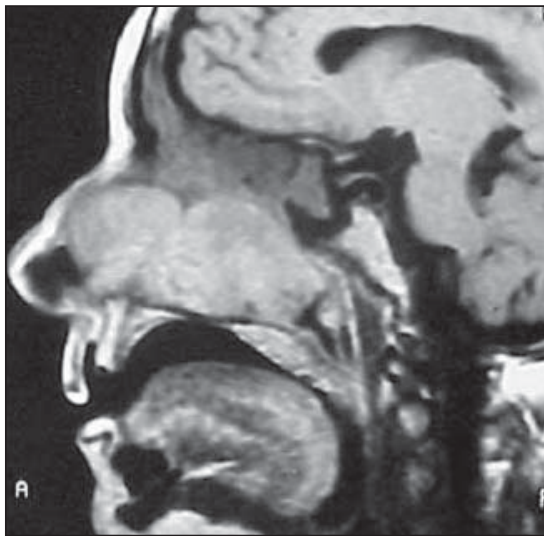

B

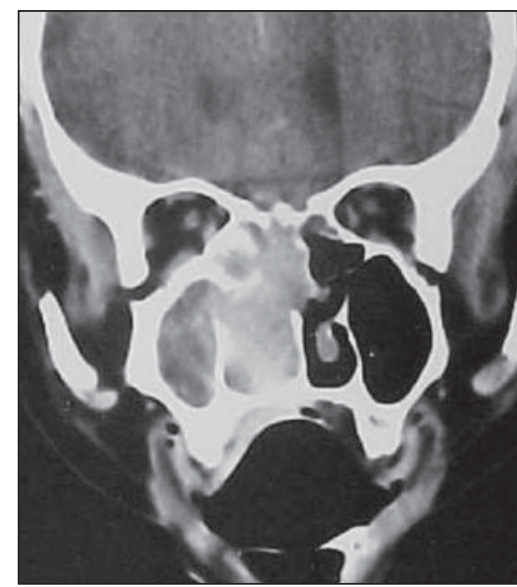

B

Figura 3. A: Tomografia computadorizada com janela para partes moles, no plano axial, evidencia lesão infiltrativa e heterogênea preenchendo a fossa nasal esquerda estendendo-se para o seio maxilar ipsilateral, condicionando destruição das paredes ósseas medial e anterior. A massa ocupa a região subcutânea da face e infiltra o septo nasal, com comprometimento contralateral. B: Ressonância magnética, plano sagital, evidencia hipersinal na seqüência ponderada em T1 sem contraste.

relatos. Anteriormente, o tumor pode protruir através do vestíbulo nasal. Medialmente, o septo nasal pode estar desviado contralateralmente ou mesmo invadido. Posteriormente, o tumor pode estender-se para a nasofaringe e, superiormente, para o seio etmoidal e para o teto da cavidade nasal ${ }^{(4,8,9)}$

Interpretação detalhada deve ser obtida na avaliação da cavidade infraorbital, fossa craniana, fossa pterigopalatina e espaço infratemporal, pois representam áreas críticas para o acesso excisional do tumor, bem como no planejamento radioterápico. A TC e a RM são importantes na avaliação prévia ao tratamento, assim como, também, na avaliação pós-tratamento ${ }^{(6)}$.
Nos casos analisados, apenas três dos 12 pacientes não mostraram nenhum tipo de extensão tumoral, ficando esses apenas confinados na cavidade nasal. Nove pacientes apresentaram extensão para um ou mais sítios, indicando estágio avançado da neoplasia no momento do diagnóstico. Os locais mais comuns de extensão tumoral foram o seio maxilar e o seio etmoidal (em seis dos 12 casos), e a cavidade nasal contralateral, lâmina crivosa do etmóide e órbita (em cinco dos 12 casos). Também houve extensão para a nasofaringe e espaço mastigatório (em dois dos 12 casos).

A TC é mais útil para avaliação da cortical óssea (lâmina crivosa, septo nasal, órbita e parede dos seios paranasais), ao 


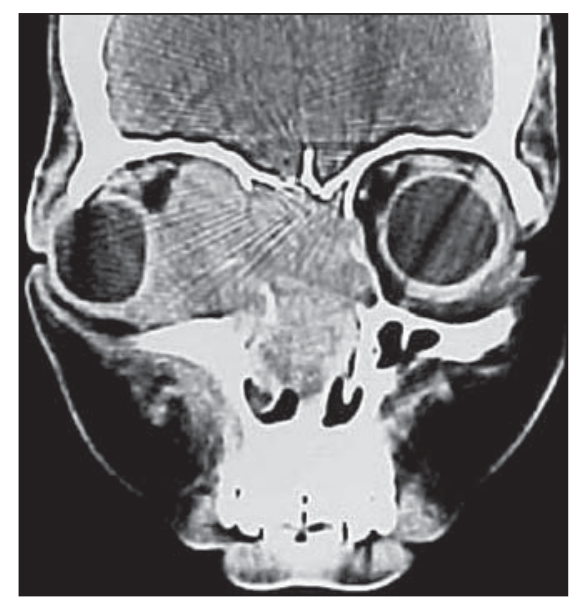

Figura 4. Tomografia computadorizada com janela para partes moles, no plano coronal, evidencia lesão infiltrativa e heterogênea ocupando a porção superior da cavidade nasal direita, estendendo-se para os seios etmoidais, fossa nasal contralateral e ocupando a órbita, provocando deslocamento lateral do globo ocular. Estesioneuroblastoma.

passo que a RM tem a vantagem de avaliar tecidos moles, a cavidade medular óssea, a base do crânio e a órbita. Embora a TC possa distinguir tumores de doenças inflamatórias, esta tarefa pode ser difícil. Com a RM isso é possível na maioria dos tumores nasossinusais (diferenciar reação inflamatória e secreções retidas da massa tumoral). Isto se deve à grande quantidade de água contida nos processos inflamatórios, resultando em aumento do sinal em T2. A maioria dos tumores nasossinusais é altamente celularizada, e portanto, apresentando sinal intermediário nas seqüências $\mathrm{T} 2^{(\mathbf{1 0}, \mathbf{1 1})}$. No presente estudo, em um dos casos analisados, a RM pôde diferenciar claramente a massa tumoral da secreção retida no seio maxilar.

\section{CONCLUSÃO}

A análise precisa da extensão local e disseminação tumoral fornecida pela TC e RM desempenha papel importante no planejamento terapêutico, influenciando também o prognóstico.

\section{REFERÊNCIAS}

1. Batsakis JD. Cancer of nasal cavity and paranasal sinuses. In: Batsakis JD, ed. Tumors of head and neck. Clinical and pathological considerations. 2nd ed. Baltimore, MD: Williams \& Wilkins, 1979: 327-43.

2. Mafee MF. Nonepithelial tumors of the paranasal sinuses and nasal cavity. Role of CT and MR imaging. Radiol Clin North Am 1993;31:75-90.

3. Maroldi R, Farina D, Battaglia G, Maculotti P, Nicolai P, Chiesa A. MR of malignant nasossinusal neoplasms. Frequently asked questions. Eur J Radiol 1997;24:181-90.

4. Tufano RP, Mokadam NA, Montone KT, et al. Malignant tumors of the nose and paranasal sinus: Hospital of the University of Pennsylvania experience 1990-1997. Am Rhinol 1999;13:117-23.

5. Bonfils P, Brasnu D, Menard M, Jaubert F, Laccourreye $\mathrm{H}$. Tumeurs des fosses nasales. Etude retrospective dune series de 67 cas. Ann Otolaryngol Chir Cervicofac (Paris) 1989;106:225-31.

6. Fava AS, Carvalho MD. Tumores malignos do nariz, cavidades nasais e seios paranasais. In: Brandão RG, Ferraz AR, eds. Cirurgia de cabeça e pescoço. São Paulo, SP: Rocca, 1989:493-505.

7. Hudgins PA. Sinonasal imaging. Neuroimaging Clin N Am 1996;6:319-31.

8. Chow JM, Leonetti JP, Mafee MF. Ephitelial tumours of the paranasal sinuses and nasal cavity. Radiol Clin North Am 1993;31:61-73.

9. Osborn AG, Mcliff EB. Computed tomography of the nose. Head Neck Surg 1982;4:182-99.

10. Hodges FG, Gado M, Sartor K. Diagnosis of tumors of paranasal sinus and nasal cavity. In: Thawley SE, Panje WR, Batsakis JG, Lindberg RD, eds. Comprehensive management of head and neck tumors. 2nd ed. Philadelphia, PA: WB Saunders, 1987: 278-303.

11. Som PM, Shapiro MD, Biller HF, Sasaki C, Lawson W. Sinonasal tumours and inflammatory tissues: differentiation with MR imaging. Radiology 1988; 167:803-8. 